\title{
"I Am the Eye in the Sky - Can You Read My Mind?" How to Address Public Concerns Towards Drone Use
}

\author{
Anne Oltvoort ${ }^{1}$, Peter de Vries ${ }^{1(\bowtie)}$, Thomas van Rompay ${ }^{2}$, \\ and Dale Rosen ${ }^{3}$ \\ ${ }^{1}$ Psychology of Conflict, Risk and Safety, \\ University of Twente, Enschede, The Netherlands \\ \{a.b.a.oltvoort,p.w.devries\}@utwente.nl \\ ${ }^{2}$ Communication Science, University of Twente, Enschede, The Netherlands \\ t.j.l.vanrompay@utwente.nl \\ ${ }^{3}$ BMS Lab, University of Twente, Enschede, The Netherlands \\ dalerosen15@gmail.com
}

\begin{abstract}
Inspired by recent debates on drone technology and privacy protection, this research examines how negative consequences of drone usage can be mitigated by tailoring information about drone employment to the environmental context in which they are used. Additionally, this study seeks to clarify the role of information needs people have when confronted with drones in different settings. Using virtual reality environments and a dedicated virtual app providing opportunities for the public to learn more about drone usage, participants were confronted with drone surveillance at either a business area, at a park, or during an event, and received transparent information on drone usage or a neutral message proving no information on drone usage. Additionally, participants could obtain more information on drone usage by clicking on one or more information buttons in the app. Results show that, compared to an event, participants were less acceptant of drones in a business area and even less so at the park. Further analyses indicated that heightened transparency perceptions resulted in higher levels of trust, perceived control, and drone acceptance. Finally, participants particularly sought information on how drones are used in the business area and park environment, whereas a need for privacy information stood out in the park context. These findings testify to the importance of careful consideration of the environmental context and related communication needs people have when informing the public about drone usage.
\end{abstract}

Keywords: Drones $\cdot$ Acceptance $\cdot$ Transparency

\section{Introduction}

Drones are steadily finding their way into everyday life. Apart from consumers using drones for entertainment purposes, drones are also increasingly deployed by a wide variety of (governmental) organizations and event organizers to improve detection and prevention of crime, and to enable enhanced data collection for incident management 
purposes [1]. Consider for instance drones used by firefighters which are equipped with cameras and sensors that are able to collect information about possible toxic substances in the air. Live video footage and information about air composition could allow firefighters to better anticipate and prepare for upcoming emergencies. Despite such evident advantages however, organisations such as local governments, police forces, and event organisers are often hesitant to deploy drones [2]. Not only may members of the public infer that something is amiss - something especially event organisers want to avoid at all cost - people might also feel that their privacy is at stake. Several recent cases indeed suggest that civilians are becoming increasingly suspicious and hostile when confronted with drones humming overhead [3], in some cases even triggering explicit acts of violence and aggression (e.g., shooting a drone from the sky [4]).

Inspired by the division between proponents of drone use, who are mainly attuned to the opportunities which drones provide for enhancing public safety and security and opponents and critics, who have pointed out aforementioned privacy concerns and weariness regarding drone usage, the current research seeks to find middle ground by proposing that concerns and fears regarding drone usage can be remedied by contextspecific information disclosure strategies. More specifically, we will argue that feelings and fears regarding drone use vary with context, and that therefore different information-disclosing strategies are needed across different types of settings. Furthermore, we seek to gain insight into information needs triggered by drone perception across environmental settings. For instance, are needs for (additional) privacy information less prevalent in settings where drones are typically common and expected (e.g., a large event where safety management is obviously an issue of concern) compared to settings where surveillance feels out of place (e.g., at a public park where people come to unwind and reboot)?

In other words, negative effects and consequences of drone usage may be remedied when tailoring information disclosure strategies to the specific information needs civilians have across different environmental settings. Hence our research question:

How does acceptance of drones vary with environmental context and what information disclosure strategy contributes to the acceptance of government's use of drones?

\section{Background}

Several negative effects of drone use have been noted across studies in recent years. Rahman, for instance, mentions 'Orwellian' fears of 'being followed' and mass surveillance, concerns over abuse or misuse of footage, and growing perceptions of ever more impersonal and distant relationships with police and law enforcers [5]. Furthermore, Custers [6] lists a number of negative effects with respect to privacy, including the 'Chilling effect', 'Function creep', and 'Privacy of location and space'. The chilling effect is a term used to describe people being more self-conscious and less free-wheeling when they know they are being watched by authorities. Function creep refers to governments initially using drones for acceptable purposes, such as a missingperson search, but gradually shifting towards more controversial purposes, such as mass surveillance. Privacy of location and space refers to the right a person has not be identified or monitored when moving in public, semi-public or private places. 
Literature suggests that the effects of drone use on safety perceptions vary with the extent to which a context is experienced as private. Indeed, Taylor [7] found people to feel less safe when filmed in private environments rather than in public places. Thus, being (almost) alone in a peaceful park may feel like a relatively private experience, and observing an 'out of place' drone overhead may negatively affect safety perceptions by signaling that something is amiss.

Other research points to the importance of people's inferences with respect to drone use. Van Rompay et al. [8], for example, showed that CCTV camera presence in a city center positively impacted participants' affective evaluation of the environment as it is interpreted as a sign of good intent. Specifically, in such an 'appropriate' setting, camera presence elicited positive inferences about law enforcers and policy makers and their intentions (e.g., "They know what is going on, they know what they are doing, and they do it with citizen safety in mind"). Similarly, Taylor [7] showed that individuals who had no difficulties in accounting for the presence of CCTV cameras ("The CCTV is there to prevent crime", p. 309) were also less likely to experience problems with their presence. On the other hand, when camera presence is not perceived as appropriate or natural (e.g., in everyday public settings where risk perceptions are low or non-existent), people have been found to behave more negatively as CCTV is interpreted as a sign of distrust [7].

Thus, when it is difficult for people to come up with logical reasons or inferences as to why drones are employed in a specific context (e.g., drones employed at a peaceful park), drones may readily inspire confusion and weariness, and may for that reason inspire distrust and an overall negative attitude. On the other hand, when drones are readily perceived as contributing to safety and security (e.g., at a large event), attitude formation takes an altogether different route and safety perceptions and feelings of wellbeing are arguably enhanced. In sum, the context in which a drone is employed might well be a crucial factor to consider when seeking to enhance public acceptance of drones. Whereas the need for information might be lower or non-existent in settings where drone usage is expected, it might be particularly important for organisations such as local governments and police units to invest in information-disclosure strategies in settings where drones are perceived as less commonplace. In these cases, transparency, i.e., informing members of the public about the true reasons behind drone use, might be essential to avert incorrect inferences and belief formation which may be detrimental for the public's acceptance of drones.

Transparency is believed to be an underlying factor in this process of acceptance, because it could (re)establish trust in organisations [9-13] and it could evoke a sense of perceived control [14]. Transparency is considered to consist of three underlying concepts: disclosure, clarity and accuracy. Disclosure is defined as the perception that relevant information is received in a timely manner [e.g., 15, 16]. This implies that information should be shared openly (without holding back) and timely. Clarity is defined as the perceived level of lucidity and comprehensibility of information received from a sender [17]. Information should be presented clearly and in a concrete (rather than overly abstract) manner by organizations for it to be transparent. Accuracy is defined as the perception that information is correct to the extent possible given the relationship between sender and receiver [17]; information cannot be seen as transparent when it is purposefully biased or unfoundedly contrived [13]. In short, 
information about drones that is timely, comprehensible, and accurate may (re)establish trust in the organisation (i.e., the sender of the communication).

Trust, in turn, plays a major role in overcoming risk perceptions and in the acceptance of new technologies [e.g., 18, 19]. Trustworthiness of an organisation is based on attributed goodwill (or: benevolence), integrity, and competence [20]. Goodwill refers to "the extent to which a trustee is believed to want to do good to the trustor, aside from an egocentric profit motive" [20, p. 718]. Integrity refers to "the trustor's perception that the trustee adheres to a set of principles that the trustor finds acceptable" [20, p. 719]. Competence refers to "the group of skills, competencies, and characteristics that enable a party to have influence within some specific domain" [20, p. 717]. Thus, effectively communicating an organisation's goodwill, integrity and competence may positively affect trust in that organisation, and, probably, acceptance of drones it deploys.

Transparent information from an organisation (e.g., about the course of implementation and by addressing possible concerns about the impact of a new technological innovation) may boost user involvement [14]; user involvement, in turn, has been shown to increase a sense of control and acceptance. Mills and Krantz [21], for instance, showed that moderate levels of choice and information provided to blood donors proved effective for coping with stress, arguably because they experienced higher levels of control over the situation.

\subsection{The Current Study}

Participants were exposed to a Virtual Reality (VR) environment - a park, business area, or a festival - and, as part of the VR scenario, watched a drone fly overhead. In VR, participants had a (virtual) smart phone with an app; transparency was manipulated by providing the option to look for information about the drone. Several menu options were provided, each representing different types of information (e.g., information on the 'why' and 'how' of drone usage and privacy information). The effects of context and transparency on trust, control and acceptance was measured. By logging the use of these menu options, we aimed to determine which kinds of information people preferred in the different environmental settings. The conceptual model is shown in Fig. 1.

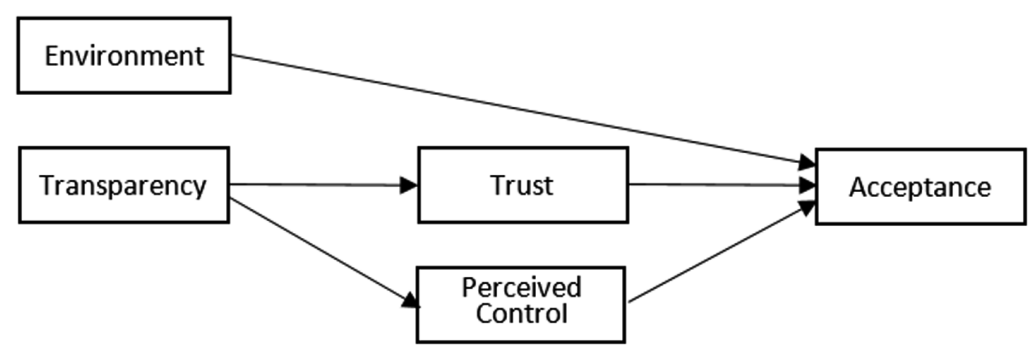

Fig. 1. Conceptual model 


\section{Method}

\subsection{Participants and Design}

120 participants $\left(69 \mathrm{~F}, 51 \mathrm{M}, M_{\text {age }}=24.30, S D=6.58\right.$, range $=19-61$ y) participated in this study. They were randomly assigned to distributed across the cells of a 2 (Transparency: yes versus no) *3 (Context: event versus business area versus park) between-participants design with acceptance as dependent variable.
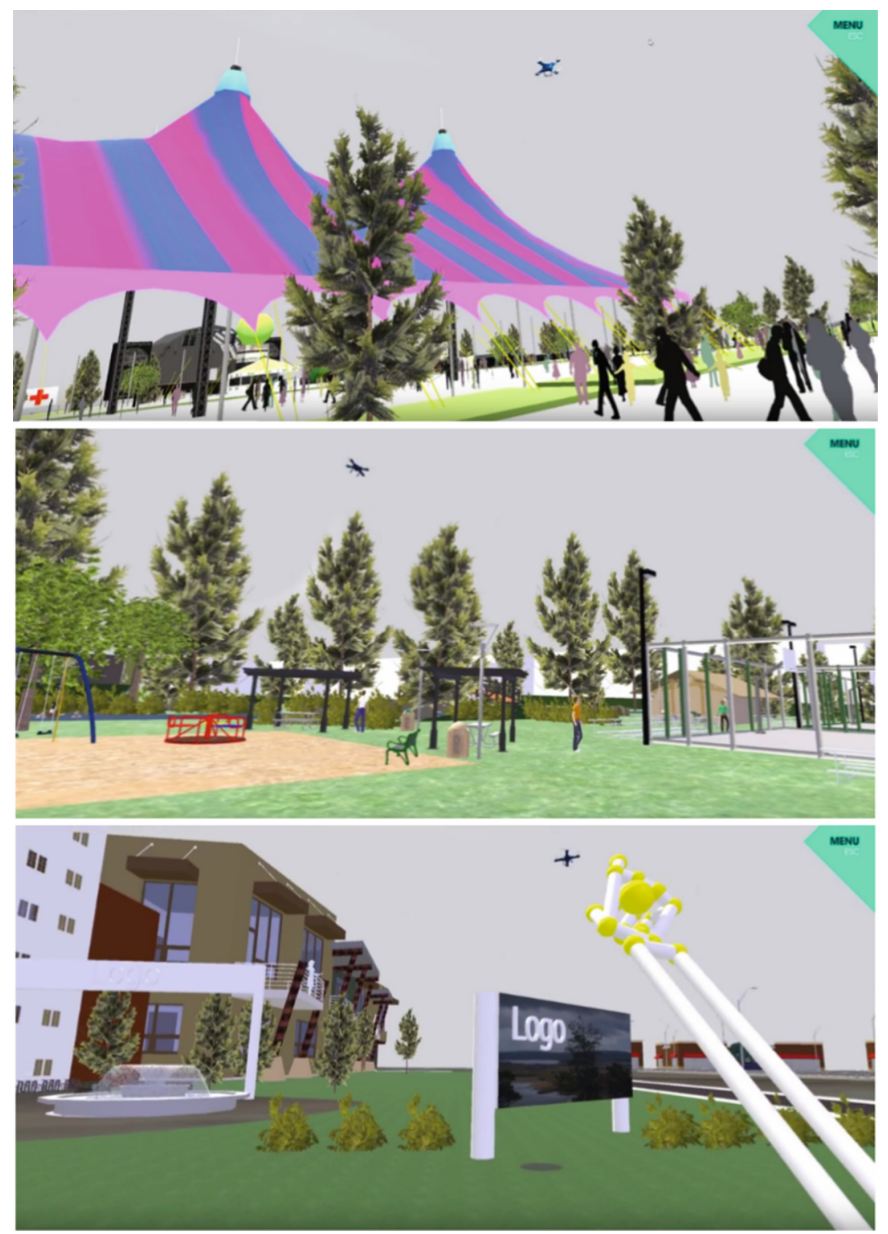

Fig. 2. Environment conditions: event (top), park (middle), and business area (bottom).

\subsection{Procedure}

First of all, participants received an introductory text about the experiment, and were assigned to one of the virtual environments. 
Environment. Participants were given a VR head set and placed in a virtual environment. The VR scenario that unfolded was either situated at an event, in a park, or at a business area. Participants were positioned in a fixed spot, where they could look around freely. After a short time they could hear and see a drone overhead; they had been informed beforehand that the drone belonged to the Enschede Municipality. Figure 2 shows screenshots of each condition.

Transparency. After $30 \mathrm{~s}$ participants received a push notification of the 'Municipality of Enschede Drone App'. In the transparent condition, this app conveyed information related to drone use. Participants could obtain this information by clicking on six menu options: 1. Who (explicating the Enschede municipality to be the organisation responsible for the drone deployment), 2. Why (making clear that drones are used to make [the specific environment] a pleasant and safe place for everyone), 3 . How (briefly explaining that security personnel will be alerted if risky situations or behaviours are detected), 4 . Privacy (underscoring that visitors' privacy is taken very seriously and that the drone is incapable of detecting actual individuals), 5. Images/map (showing what kind of footage is collected with the drone, and where), and 6. Feedback (offering the possibility to ask questions or give feedback).

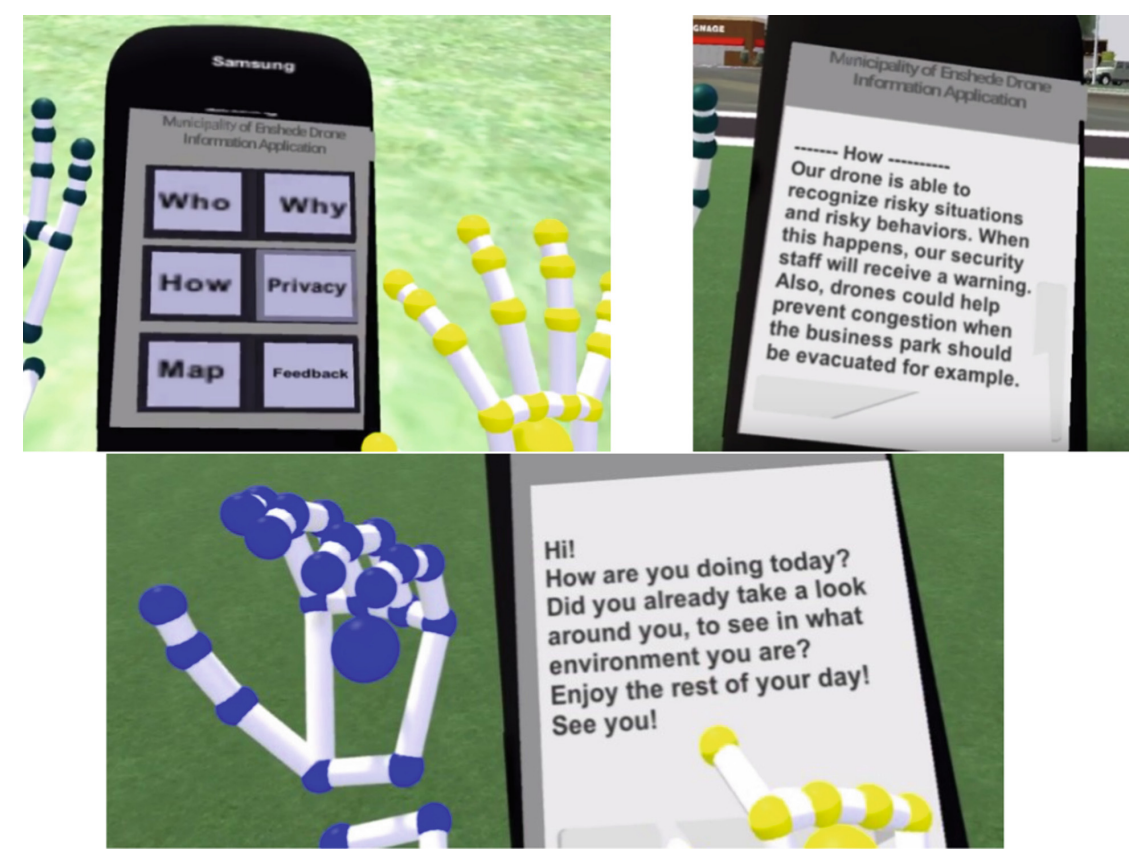

Fig. 3. Transparency conditions: transparency (top row) and no transparency (bottom)

Participants could click on as many options as they wanted. Their choices and the time they spent reading the specific information were logged. In the control condition (no transparency) participants did not receive a push notification of the 'Municipality of 
Enschede Drone App', but instead received a neutral message (e.g., 'Hi! How are you doing today? Did you already take a look around you, to see in what environment you are?'). After approximately $2 \mathrm{~min}$ the smart phone disappeared; subsequently, the drone appeared in the sky and flew over the terrain for about $2 \mathrm{~min}$. Figure 3 shows the Transparency conditions.

After answering the questions pertaining to the dependent variables, participants were debriefed and thanked.

Materials. The materials for this experiment were created with the help of the University of Twente's BMS Lab. The three environments were created in 3D; characters were built with Reallusion Iclone7 and Character Creator 2. Oculus CV1 was used to immerse participants in the VR environments.

\section{Measures}

Perceived Transparency. The level of Perceived Transparency (regarding the Municipality of Enschede), was measured using a 7-point Likert scale (ranging from 1 = strongly disagree to 7 = strongly agree), using items from Rawlings [22]. Participants rated their level of agreement on four items such as "The municipality of Enschede wants to understand how its decisions affect people like me" (Cronbach's $\alpha=.69$; Guttman's $\lambda_{2}=.70$ ).

Trust. To determine the participant's level of Trust in the organisation thirteen items from Rawlings [22] were used, using a 7-point Likert scale (ranging from $1=$ strongly disagree to 7 = strongly agree). A distinction was made between the three dimensions of trust (goodwill, integrity and competence) and overall trust. Goodwill was measured with three items (e.g., "I believe the municipality of Enschede takes the opinions of people like me into account when making decisions"), Integrity with four items (e.g., "The municipality of Enschede treats people like me fairly and justly"), and Competence with three items (e.g., "I feel very confident about the skills of the municipality of Enschede"). Overall trust was measured with three items (e.g., "I trust the municipality of Enschede to take care of people like me"; $\alpha=.87$ and $\lambda_{2}=.88$ ).

Perceived Control. Perceived control was measured with five items, based on items from Ouwehand, De Ridder and Bensing [23], on a 10-point Likert scale (1 = Not at all to $10=$ A great deal). A sample item is "To what extent did you feel you could predict the situation?" $\left(\alpha=.74\right.$ and $\left.\lambda_{2}=.75\right)$.

Acceptance. The Acceptance Scale [24] was slightly adjusted to reflect the extent to which participants accepted government's use of drones, using nine Likert items (e.g., "My judgements of the drone of the municipality of Enschede is are...: Pleasant Unpleasant" $\left(\alpha=.86\right.$ and $\left.\lambda_{2}=.88\right)$.

In addition, participants were asked to indicate to what extent they considered drone usage appropriate and understandable at an event, at a park, and in a business area. Five questions of demographic data were asked (age; gender; level of education; residence; frequency of visiting Enschede). 


\section{Results}

\subsection{Effects of Context and Transparency on Acceptance}

A Multivariate Anova was conducted, with Context and Transparency as independent variables and Perceived transparency, Trust, Perceived control and Acceptance as dependent variables. The results showed non-significant main effects of Context $(F$ (8, $222)=0.68$, ns., Wilks' Lambda $=.95$ and Transparency $(F(4,111)=0.90$, ns., Wilks' Lambda $=.97)$. Also, no significant interaction was found $(F(8,222)=0.98$, ns., Wilks' Lambda $=.93)$. The lack of effect of the Transparency manipulation on Perceived transparency clearly shows that the manipulation did not produce the desired result. We therefore decided to proceed our analyses in a more exploratory manner, using Perceived transparency as the independent variable.

\subsection{Mediation Analysis}

A mediation analyses was conducted to exploratively test whether the relationship between Perceived transparency and Acceptance can be explained by Trust and/or Perceived control. Figure 4 shows the result of this mediation analysis. As can be seen here, the initial significant direct effect of Perceived transparency on Acceptance (showing that perceived transparency increased acceptance; $B=0.32, p<.005$ ) was reduced to insignificance $(B=-0.02$, n.s.) when the proposed mediators Trust and Perceived Control were added to the model. Subsequent Sobel tests show that both indirect paths (i.e., via Trust and Perceived control) are significant (Trust: Sobel $z=3.64, p<.001$; Perceived control: Sobel $z=2.59, p=.010)$. These results suggest that the effect of Perceived transparency on Acceptance are mediated by both Trust and Perceived control.

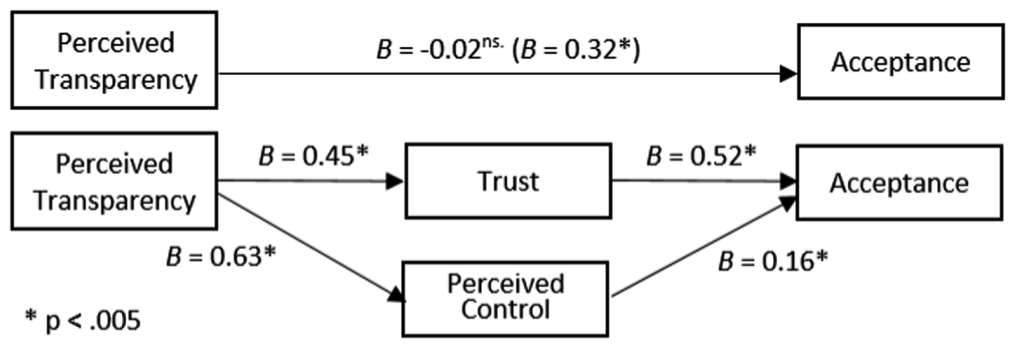

Fig. 4. Mediation analysis

\subsection{Effects of Environment on Acceptance}

Participants were asked to indicate the extent to which they considered drone usage appropriate and reasonable in different contexts (event, business area and park). A repeated measures Anova was conducted to compare the acceptance rates among the three contexts. The analysis showed the contexts differed significantly from each other, 
$F(2,118)=121.08, p<.001$, Wilks' Lambda $=.33$. Pairwise comparisons indicated drones were significantly more accepted during events compared to business areas $\left(\mathrm{M}_{\text {difference: }} 1.58, S E=0.13, p<.001\right)$ and compared to parks $\left(M_{\text {difference }}=2.01\right.$, $S E=0.13, p<.001)$. Drones were also significantly more accepted at business areas compared to parks $\left(M_{\text {difference }}=0.43, S E=0.12, p=.001\right)$.

\subsection{Effects of Environment on Information Use}

The need for information participants experienced (as a function of the context in which the drone appeared), was analyzed with a multivariate repeated-measures Anova with the time spent reading the information provided by each of the six app buttons as dependent variables and Environment as independent variable. This resulted in a significant main effect of Environment, $F(12,106)=2.44, p=.008$, Wilks' Lambda $=.609$. Univariate analyses revealed effects of Environment on How $(F(2,57)=5.66, p=.006)$ and Privacy $(F(2,57)=6.13, p=.004)$.

Follow-up analyses with the time spent reading 'How' information revealed a significant difference between the Event and Park condition: in the former participants spent more time reading the information than in the latter $(M=11.82, S D=8.72$ versus $M=3.26, S D=5.26, p=.002)$. Additionally, in the Business area condition reading times were higher than in the Park condition $(M=8.91, S D=9.85$ versus $M=3.26, S D=5.26, p=.033)$. No differences were found between the Event and Business area conditions $(\mathrm{p}=.264)$. Apparently, both in the Event and the Business area condition participants were interested in finding out how the drone operated. Reversely, analyses relating to the time spent reading Privacy information showed that the need for this information was lower in the Event than in the Park condition $(\mathrm{M}=10.67, \mathrm{SD}=10.35$ versus $M=20.53, S D=15.74, p=.013)$. This need was also lower in the Business area condition compared to the Park condition $(M=7.58$, $S D=9.61$ versus $M=20.53, S D=15.74, p=.001)$. Hence, in the park condition, the need for privacy information stood out. No difference was found between the Event and Business area conditions $(p=.427)$.

\section{Conclusions and Discussions}

The current study examined whether the acceptance of drones differs among contexts and whether transparent information disclosure increases acceptance of drones. Although direct manipulations of context and transparency failed, more explorative analyses did provide useful insights. First, the degree to which people thought drone deployment was appropriate or reasonable was shown to vary between contexts: drones were more acceptable during events compared to business areas and to parks, and more acceptable at business areas compared to parks.

Second, perceived (rather than manipulated) transparency proved to have positive effects on trust in the organization utilizing the drones (i.e., the municipality), on the degree of control they perceived to have, and on the willingness to accept drones. Additional analyses showed that, in conformance with expectations, the relationship between transparency and acceptance can be explained by trust and perceived control. 
It should be noted however that these findings are purely correlational, necessitating cautious interpretation of the causality of these relationships.

Interestingly, the need for information participants experienced also depended on the context in which the drone appeared, especially information pertaining to 'privacy' and to 'how' the drone and the system behind it functioned. For both types of information, presented after pushing the respective app buttons, the event and business area contexts stood out against the park context. In the former two contexts, the time spent reading how-information was higher and time spent reading privacy information was lower than in the latter. The findings relating to privacy tie in with literature about effects of CCTV. Taylor [7], for instance, found people to feel less safe when filmed in private environments than in public places and have a higher need for information remedying such negative feelings. By extension, being at a park may feel like a relatively private experience, and observing a drone overhead may inspire feelings of being watched and related privacy concerns, resulting in an enhanced need for privacy information. The event and business area contexts were likely judged as less private (and drone presence as more appropriate), which may have reduced participants' need for privacy information. As to why reading times for the 'how' of drone usage were higher in the event and business area conditions (as opposed to the park condition), our results do not provide a straightforward answer. Perhaps, when drone presence comes across as appropriate, reasonable and for the safety of all, people are intrinsically motivated to learn more about drones, and hence may more readily click the 'How' button. In the park context where fears and privacy concerns take centre stage, a corresponding inclination to click on the 'Privacy' button may transpire.

Because of various practical problems connected to real-life drone use for research purposes, we decided to administer the scenarios in Virtual Reality. One may argue that this would confront participants with inherently artificial environments, and that this likely leads to artificial findings. In answer to this we would like to point out that there are quite some studies providing convincing support for ecological validity of scenario studies in general [25, 26], and studies employing Virtual Reality specifically [27]. After explicitly comparing the experiential qualities of real and VR environments, Kuliga et al. [27] concluded VR to have strong potential to be used as an empirical tool in psychological research.

Having said so, it is of course true that VR environments (including ours) usually lack fully immersive atmospherics, social dynamics, and multi-sensory stimulation which are typical of real-life settings and of events in particular (where people gather partly because of social dynamics and sensations of many kinds). Although as such, these are shortcomings of our VR manipulation, in all likelihood enriching future VR encounters along these lines could be expected to lead to even stronger effects. Hence, future research could involve more realistic virtual environments, for instance with more detailed graphic rendering, additional environmental sounds, more people moving about, and incorporate an actual rather than a virtual smartphone app. This way, future research could also incorporate more realistic and subtle ways of informing people by, for instance, automatically sending a message to people's phones who are near a drone [cf. 28]. 
In conclusion, our findings underscore the importance of being responsive to the needs and values of specific target audiences while communicating about drone usage [cf. 29]. Findings of the current research may provide a first step to compose effective communication and provide indications as to what specific information needs should take precedence in which settings.

\section{References}

1. Winkler, S., Zeadally, S., Evans, K.: Privacy and civilian drone use: the need for further regulation. IEEE Secur. Priv. 16, 72-80 (2018)

2. de Vries, P., Galetzka, M., Gutteling, J.: Persuasion in the wild: communication, technology, and event safety. In: Spagnolli, A., Chittaro, L., Gamberini, L. (eds.) PERSUASIVE 2014. LNCS, vol. 8462, pp. 80-91. Springer, Cham (2014). https://doi.org/10.1007/978-3-31907127-5_8

3. Jolly, J.: 'Never, ever try to shoot at a drone.' Neighborhoods buzz with complaints over pesky drones. USA Today (2018). https://eu.usatoday.com/story/tech/columnist/2018/09/03/ drone-gripes-mount-homeowners-complain-breached-privacy-annoyance/1117085002/

4. Witteman, J.: Wanneer schendt een drone uw privacy? De Volkskrant (2017). https://www. volkskrant.nl/cultuur-media/wanneer-schendt-een-drone-uw-privacy- b91d9c53/

5. Rahman, M.F.A.: Security Drones: is the Singapore Public Ready? (2016)

6. Custers, B. (ed.): The Future of Drone Use: Opportunities and Threats from Ethical and Legal Perspectives. ITLS, vol. 27. T.M.C. Asser Press, The Hague (2016). https://doi.org/10. 1007/978-94-6265-132-6

7. Taylor, E.: I spy with my little eye: the use of CCTV in schools and the impact on privacy. Sociol. Rev. 58, 381-405 (2010)

8. Van Rompay, T.J.L., De Vries, P.W., Damink, M.T.: "For your safety": effects of camera surveillance on safety impressions, situation construal and attributed intent. In: MacTavish, T., Basapur, S. (eds.) PERSUASIVE 2015. LNCS, vol. 9072, pp. 141-146. Springer, Cham (2015). https://doi.org/10.1007/978-3-319-20306-5_13

9. Bennis, W., Goleman, D., O’Toole, J.: Transparency: How Leaders Create a Culture of Candor. Wiley, Hoboken (2008)

10. Fombrun, C.J., Rindova, V.P.: The road to transparency: reputation management at Royal Dutch/Shell. Expressive Organ. 7, 7-96 (2000)

11. Jahansoozi, J.: Organization-stakeholder relationships: exploring trust and transparency. J. Manag. Dev. 25, 942-955 (2006)

12. Tapscott, D., Ticoll, D.: The Naked Corporation: How the Age of Transparency Will Revolutionize Business. Simon and Schuster, New York City (2003)

13. Walumbwa, F.O., Avolio, B.J., Gardner, W.L., Wernsing, T.S., Peterson, S.J.: Authentic leadership: development and validation of a theory-based measure. J. Manag. 34, 89-126 (2008)

14. Baronas, A.-M.K., Louis, M.R.: Restoring a sense of control during implementation: how user involvement leads to system acceptance. MIS Q. 12, 111-124 (1988)

15. Bloomfield, R., O'Hara, M.: Market transparency: who wins and who loses? Rev. Financ. Stud. 12, 5-35 (1999)

16. Clark Williams, C.: Toward a taxonomy of corporate reporting strategies. J. Bus. Commun. 1973(45), 232-264 (2008)

17. Schnackenberg, A.K., Tomlinson, E.C.: Organizational transparency: a new perspective on managing trust in organization-stakeholder relationships. J. Manag. 42, 1784-1810 (2016) 
18. Gefen, D., Karahanna, E., Straub, D.W.: Trust and TAM in online shopping: an integrated model. MIS Q. 27, 51-90 (2003)

19. Pavlou, P.A., Gefen, D.: Building effective online marketplaces with institution-based trust. Inf. Syst. Res. 15, 37-59 (2004)

20. Mayer, R.C., Davis, J.H., Schoorman, F.D.: An integrative model of organizational trust. Acad. Manag. Rev. 20, 709-734 (1995)

21. Mills, R.T., Krantz, D.S.: Information, choice, and reactions to stress: a field experiment in a blood bank with laboratory analogue. J. Pers. Soc. Psychol. 37, 608 (1979)

22. Rawlins, B.R.: Measuring the relationship between organizational transparency and employee trust. Public Relat. J. 2, 1-21 (2008)

23. Ouwehand, C., De Ridder, D.T.D., Bensing, J.M.: Situational aspects are more important in shaping proactive coping behaviour than individual characteristics: a vignette study among adults preparing for ageing. Psychol. Health 21, 809-825 (2006)

24. Van Der Laan, J.D., Heino, A., De Waard, D.: A simple procedure for the assessment of acceptance of advanced transport telematics. Transp. Res. Part C: Emerg. Technol. 5, 1-10 (1997)

25. Bateson, J.E., Hui, M.K.: The ecological validity of photographic slides and videotapes in simulating the service setting. J. Consum. Res. 19, 271-281 (1992)

26. Stamps III, A.E.: Use of photographs to simulate environments: a meta-analysis. Percept. Mot. Skills 71, 907-913 (1990)

27. Kuliga, S.F., Thrash, T., Dalton, R.C., Hölscher, C.: Virtual reality as an empirical research tool-exploring user experience in a real building and a corresponding virtual model. Comput. Environ. Urban Syst. 54, 363-375 (2015)

28. Thomasen, K.: Beyond Airspace Safety: A Feminist Perspective on Drone Privacy Regulation (2017)

29. PytlikZillig, L.M., Duncan, B., Elbaum, S., Detweiler, C.: A drone by any other name: purposes, end-user trustworthiness, and framing, but not terminology, affect public support for drones. IEEE Technol. Soc. Mag. 37, 80-91 (2018) 